\title{
Proposed few-optical cycle laser-driven particle accelerator structure
}

\author{
T. Plettner, P. P. Lu, and R. L. Byer \\ E.L. Ginzton Laboratories, Stanford University, Stanford, California 94305, USA
}

(Received 2 October 2006; published 14 November 2006)

\begin{abstract}
We describe a transparent dielectric grating accelerator structure that is designed for ultrashort laser pulse operation. The structure is based on the principle of periodic field reversal to achieve phase synchronicity for relativistic particles; however, to preserve ultrashort pulse operation it does not resonate the laser field in the vacuum channel. The geometry of the structure appears well suited for application with high average power lasers and high thermal loading. Finally, it shows potential for an unloaded gradient of $10 \mathrm{GeV} / \mathrm{m}$ with $10 \mathrm{fs}$ laser pulses and the possibility to accelerate $10^{6}$ electrons per bunch at an efficiency of $8 \%$. The fabrication procedure and a proposed near term experiment with this accelerator structure are presented.
\end{abstract}

DOI: 10.1103/PhysRevSTAB.9.111301

PACS numbers: 41.75.Jv, 41.75.Ht, 42.25.Bs

\section{INTRODUCTION}

\section{A. Background}

Ultrashort pulse few-optical cycle tabletop lasers have become a reality in recent years [1,2]. An important factor for this realization has been the development of carrierenvelope stabilization techniques [3-5] that have not only led to the ability to achieve such few-cycle pulses but also for the first time allow for the precise control of the optical phase within the laser pulse [6], which opens the possibility to construct phase-coherent mode-locked laser arrays [7]. This development of ultrafast laser technology is of fundamental importance for future laser-driven particle accelerators.

Application of such short pulses for laser-driven particle accelerators appears especially appealing from a gradient and an efficiency point of view. The shorter pulses enable the structure to sustain higher peak electric fields and also improve the overlap of the laser and the electron and therefore lead to higher efficiencies. The key objective is to envision an accelerator structure geometry that is naturally matched for usage with such few-cycle laser pulses and that can also maximize the average gradient from the input optical field.

Waveguide based accelerators are designed to satisfy the speed-of-light phase velocity condition for the traveling wave that is an accelerating mode. However, due to the natural group-velocity dispersion of the structure, the speed of the pulse envelope is seriously compromised and is considerably lower than $c$. Typical group-velocity values found with photonic band gap accelerators are on the order of $\mathrm{v}_{g} \sim \frac{1}{5}-\frac{1}{2} c[8,9]$. Unfortunately, these low group-velocity values result in a rapid slippage of the pulse envelope over a finite waveguide distance that scales with and is not much longer than the laser pulse length itself. Therefore operation of waveguide accelerator structures with few-cycle laser pulses will be challenging and very likely not feasible. As an alternative that practically eliminates the group-velocity problem, one could consider semi-open accelerator structures [10]. However, the large mode volume occupied by the free-space propagating laser field naturally leads to a very low overlap with the electron beam and consequently to a poor efficiency. In semi-open accelerators and waveguide structures, the driving electromagnetic field copropagates with the electron beam. Because of the transverse nature of electromagnetic waves, only a relatively small fraction of the total electric field can be oriented along the direction of propagation of the electron beam. Therefore the typical gradient of these systems is about an order of magnitude smaller than the applied electric field amplitude. One serious problem for waveguide accelerator structures is that these are usually not single mode. In addition to the desired speed-of-light phase velocity accelerating mode, additional nonaccelerating modes or non-speed-of-light modes can propagate in the waveguide and draw energy from the desired mode. This presents a challenge for coupling the electromagnetic wave into the waveguide without exciting the undesired nonaccelerating modes.

A third and more promising possibility is offered by a class of resonant closed structure devices that rely on the principle of periodic electric field modulation. The microstructure is periodic along the electron beam channel and produces a near-field periodic phase reset of the electromagnetic wave, allowing for phase synchronicity between the moving particle and the electromagnetic field. This scheme does not suffer from the group-velocity limitation and by being a closed, wavelength-sized geometry keeps the field mode volume well matched to the electron beam and thus leads to a higher structure impedance. In addition, these structures can be side pumped by a plane wave at normal incidence to the electron beam, allowing for a larger fraction of the applied electric field amplitude to produce a gradient and to significantly simplify the cou- 
pling of laser power into the structure. Accelerator structures of this type have been proposed for use with picosecond lasers and their intrinsic advantage for mitigating transverse wakefield and space charge instabilities has been investigated in the past $[11,12]$. However, due to their resonator nature they have a many-cycle field-buildup time and are not very well suited for operation with ultrashort laser pulses that are of interest to us.

\section{B. Proposed periodic field reversal structure}

Described here is a single-pass dielectric laseraccelerator structure that is based on the general principle of periodic electric field reversal. However, the key idea is that the field is not resonated in the vacuum channel, but instead is allowed to reform as a plane wave at the opposite grating and to leave the vacuum gap. The structure is twodimensional and can be constructed from two opposing dielectric binary gratings transparent for the chosen wavelength.

Figure 1 illustrates the proposed structure and shows the cross section of the geometry. Each grating pillar acts as an optical phase-delay plate that is chosen to add a $\pi$-phase shift with respect to the field in the adjacent vacuum space. This produces the desired periodic modulation of the electric field inside the vacuum channel from an incoming plane wave. The opposite grating is located in the near field and can therefore largely reconstruct the plane wave at the exit face of the structure by adding an additional $\pi$-phase shift to the reversed field regions of the vacuum gap. In accordance with the Lawson-Woodward theorem [13], it is the nonpropagating near field that provides the synchronous accelerating field and not the incoming freespace wave. This double-grating geometry bears some resemblance in its topology to the restricted periodic structures explored by Palmer [14], with the fundamental difference that the acceleration from this structure is not due to trapped cavity modes. As indicated in Fig. 1, extended overlap of a speed-of-light electron bunch in the structure with the few-cycle laser pulse envelope can be readily accomplished by well-known and simple to implement pulse-front tilt methods $[15,16]$. For a speed-of-light particle, a $45^{\circ}$ pulse-front tilt of the incoming plane wave is required.

Heat dissipation is a critical but unfortunately widely ignored aspect of any future high-luminosity and highgradient accelerator microstructure that concentrates laser and electron power in a very small region. In essence, the structure proposed here is a 2-dimensional-slab geometry, which has proven to be ideal for effective heat removal with high-power laser systems that present a similar problem of high power in a very reduced volume [17-19]. Also, since the incoming plane wave is orthogonal to the electron channel, the structure can be thin and thus drastically reduce the thermal lens or the onset of nonlinear effects such as Brillouin or Raman scattering commonly found in high-peak power waveguide devices.

In summary, from this introduction the prospect for such a structure to operate with few-cycle lasers appears very appealing and in the author's view deserves closer inspection. Section II of this article presents a quantitative field evaluation for this structure and a first-cut optimization for high gradients and high structure impedance. Section II F includes an analysis for thermal loading of the structure and the corresponding possible heat removal schemes. Finally, Sec. III describes an existing nanofabrication method and presents a proposed near-future electron beam experiment with a quartz double-grating structure.

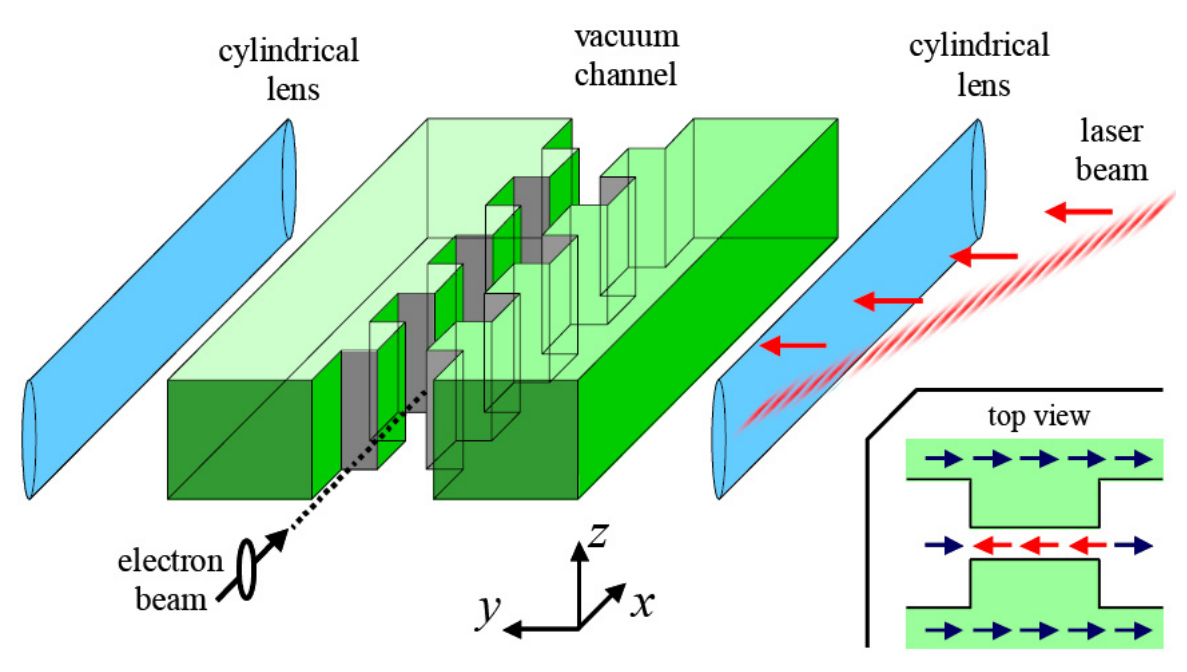

FIG. 1. (Color) Perspective view of the proposed accelerator structure. The incoming electromagnetic wave suffers a periodic phase inversion as it enters the vacuum channel. The inset shows the electric field modulation in the vacuum channel region. As the electromagnetic wave exits the vacuum channel, it experiences an additional periodic phase modulation that largely reconstructs the initial profile of the incoming wave. 


\section{EVALUATION OF THE ELECTROMAGNETIC FIELD IN THE STRUCTURE}

\section{A. Numerical evaluation method}

The 2-dimensional geometry of the structure and the choice of a plane wave at the input face are ideally suited for evaluation with well-established finite-difference time domain numerical methods (FD-TD) $[20,21]$ that evaluate the electromagnetic field components in the vacuum channel and at the exit face. The numerical analysis only requires one grating period and can use periodic boundary conditions at the sides of the region of interest and absorbing boundary conditions at the input and exit planes. For a 2-dimensional geometry two independent transverseelectric and transverse-magnetic field solutions exist. Of interest to us is an input plane wave with the electric field component polarized along the electron beam axis. This wave belongs to the transverse-magnetic field solution, whose field components evolve according to

$$
\begin{gathered}
d_{t} B_{z}=d_{y} E_{x}-d_{x} E_{y} \quad d_{t} E_{x}=d_{y} B_{z} / \mu \varepsilon \\
d_{t} E_{y}=-d_{x} B_{z} / \mu \varepsilon .
\end{gathered}
$$

The first of the three equations corresponds to the $z$-component of Faraday's law $\nabla \times \vec{E}=-d_{t} \vec{B}$ and the two latter equations correspond to Ampere's law $\nabla \times \vec{H}=$ $d_{t} \vec{D}$. No free currents or free charges are assumed in the present situation. To compute the evolution of the field components of Eq. (1), the area of interest is divided into a grid containing the instantaneous field values $E_{x}(t)$, $E_{y}(t), B_{z}(t)$ and the local dielectric constant value $\varepsilon$. In the vacuum channel $\varepsilon(\vec{r})=\varepsilon_{0}$ and in the substrate with an index of refraction $n$ the dielectric constant is $\varepsilon(\vec{r})=\varepsilon_{0} n^{2}$. The grid spacing $\Delta s$ is chosen to be much smaller that the wavelength $\lambda$. The evolution of the field components is found by evaluating Eq. (1) in discrete time and space steps

a)

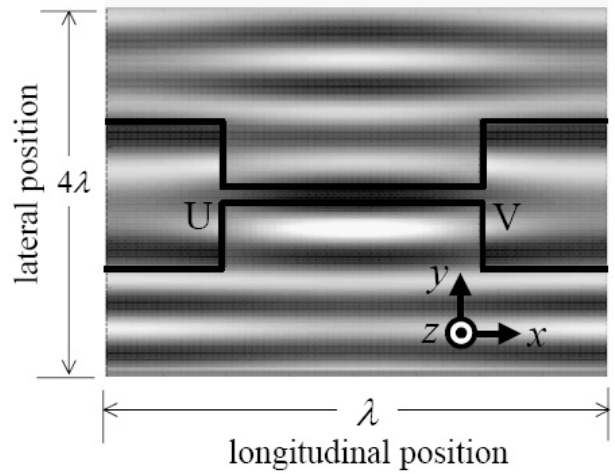

as prescribed by the particular FD-TD method employed here.

The cross-sectional area utilized for the numerical analysis is of $\lambda \times 4 \lambda$, and the mesh has a coarseness of $\lambda / 80$. This mesh resolution was tested with simple geometries having known analytical solutions for the fields, and was found to adequately model the electromagnetic field evolution. The FD-TD analysis for a structure of this type reveals that in addition to the periodic phase modulation there is an amplitude modulation in the vacuum channel, the amplitude being much larger in the narrow region of the vacuum channel than in the wide area. Figure 2(a) shows a snapshot of the electric field component parallel to the vacuum channel of a crystal quartz structure. The input plane wave is launched from the bottom and is absorbed at the top boundary. Figure 2(b) shows the maximum electric field amplitude at the surface of the grating pillar. It shows that the field is highest at the pillar center but is considerably lower near the sharp edges labeled $U$ and $V$ in Fig. 2. This is desirable because the damage threshold is expected to be lower at the sharp edges of the structure. The concentration of field at the pillars is a result of diffraction. Since the field tends to flow from the low to the high index region, the grating pillars have a focusing effect. Notice that due to diffraction the plane-wave reconstruction is not perfect and a residual amplitude and phase modulation of the exiting wave can be appreciated in Fig. 2(a).

\section{B. Estimate of the gradient}

The periodic field reversal ensures that electrons traveling in the vacuum channel experience net energy gain. The structure is designed for ideal speed-of-light particles. For relativistic electrons where $\beta$ is close to unity, a slight correction on the phase front orientation of the plane wave can maintain the extended phase synchronicity over many grating periods. The FD-TD method yields the field

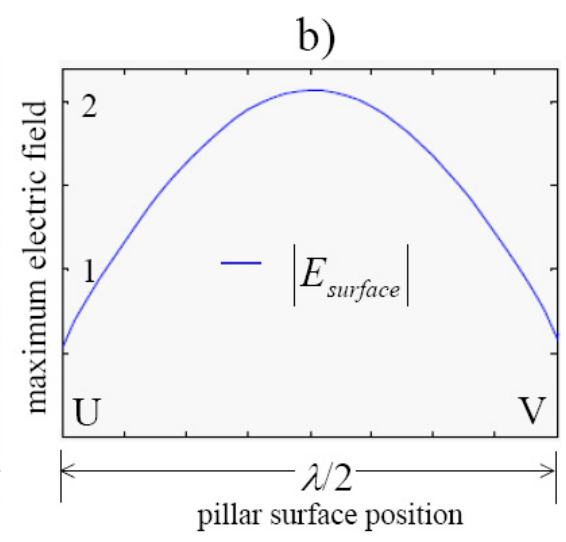

FIG. 2. (Color) (a) Gray-scale map of a snapshot of the electric field produced by an incident cw plane wave in one grating segment of the accelerator structure. The black line indicates the material-to-vacuum interface. Points $\mathrm{U}$ and $\mathrm{V}$ indicate the corners of the grating pillar, which in this example has a length of $0.9 \lambda$. The plane wave has an amplitude $E_{0}=1$ and is launched from the bottom and travels upwards. Notice the phase shift between the narrow and the wide gap regions in the vacuum channel and the reconstruction of the plane wave in the upper region. (b) The maximum surface field between points $U$ and V. 
components as functions of position and time, which allows for a straightforward calculation for the unloaded gradient and deflection forces experienced by a test particle traversing the structure. The gradient produced by the structure is the average longitudinal electric field experienced by the electron in one grating period:

$$
G_{0}=\left\langle E_{x}\right\rangle=\int_{0}^{\lambda} E_{x}[x(t), t] d x / \lambda .
$$

$E_{x}[x(t), t]$ is the longitudinal electric field at the channel center and $x(t)$ is the position of the test particle in the vacuum channel at time $t$. The field at the center of the channel $E_{x}[x(t), t]$ is obtained by the FD-TD method. Figure 3(a) shows a map of this electric field component as a function of the position at the center of the vacuum channel and time. The vertical dimension of the map corresponds to one optical cycle of the field $\tau_{\mathrm{L}}$, which as previously observed in Fig. 2(a) is mostly concentrated in the narrow region of the vacuum channel. The darker areas correspond to a decelerating field while the bright areas correspond to an accelerating field. The solid black line A shows the world line of a speed-of-light particle at the optical phase of maximum acceleration and the solid white line $\mathbf{D}$ the optical phase for maximum deceleration, which is half a cycle away from $\mathbf{A}$. The dashed black line $\mathbf{O}$ represents an optical phase for zero energy gain. Figure 3(b) shows the corresponding electric fields $E_{x}[x(t), t]$ experienced by the speed-of-light test particles traveling down the vacuum channel center. For the particular optical phases of particles $\mathbf{A}$ and $\mathbf{D}$ the average accelerating field is clearly nonzero.

For the binary grating structure of a fixed material, the free parameters that allow for gradient optimization are the aspect ratio of the grating pillar and the width of the vacuum channel. We seek to optimize these for the largest average electron energy gradient and the widest possible vacuum channel. Figure 4(a) shows the unloaded gradient as a function of grating pillar height for the same quartz substrate of index $n=1.55$ employed in the earlier examples. It can be observed that for pillar heights that approach zero there is no significant field modulation and therefore no significant gradient. The maximum attainable gradient occurs at a grating pillar height of about $0.9 \lambda$, with a value close to $\frac{1}{2}$ the amplitude of the incident laser field. As can be appreciated in the same figure, if the grating pillar is made longer the gradient drops until it reaches a minimum, corresponding to a phase difference of $2 \pi$ of the longitudinal field between the narrow and the wide parts of the vacuum channel. However, because of diffraction the cancellation of the gradient is not perfect. Figure 4(b) shows the effect of increasing the vacuum channel gap on the energy gradient. For channel gaps of $\lambda / 4$ and narrower, the gradient remains almost unchanged at a value of $G \sim 0.49 E_{0}$, where $E_{0}$ is the electric field amplitude of the input plane wave. For wider channel gaps the gradient starts to drop. This is expected since the field modulation has a feature size $\lambda / 2$, which due to diffraction does not propagate into the far field. Further widening of the channel to a width of $\lambda$ lowers the average gradient to approximately one-half the original value. Extending the gap width further is not feasible if the high gradient is to be preserved.

To find the maximum gradient, one has to find the magnitude of the largest electric field found in the structure, which should not exceed the local peak laser fluence at damage threshold. We define the damage factor as the ratio of the unloaded gradient divided by the local maximum electric field anywhere in the structure: a)

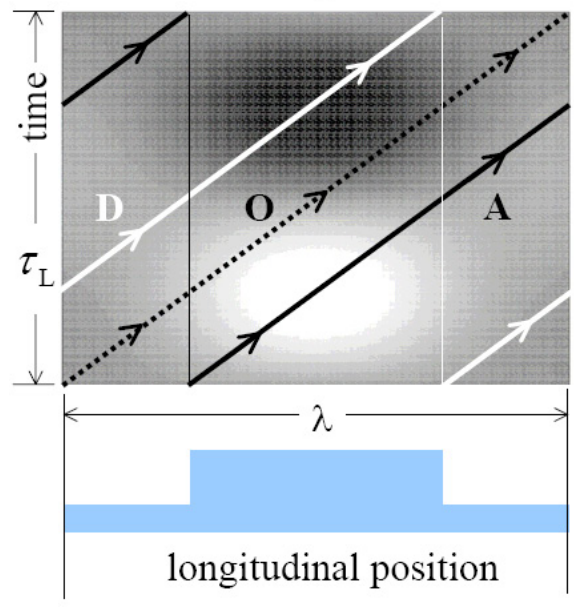

b)

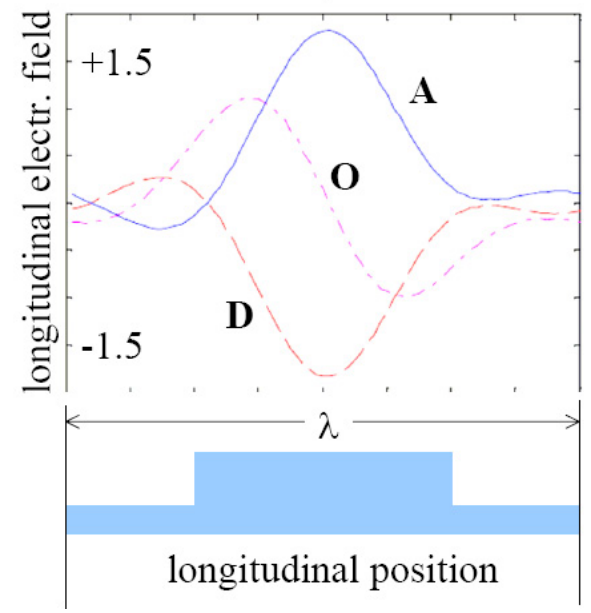

FIG. 3. (Color) (a) Map of the accelerating electric field component as a function of elapsed time and position at the center of the vacuum channel. The lines indicate the trajectory of a relativistic particle at an accelerating phase $\mathbf{A}$, a decelerating phase $\mathbf{D}$, and a phase of no energy change $\mathbf{O}$. (b) The longitudinal electric field component along these particle trajectories down the center of the vacuum channel. The electric field magnitude of these traces corresponds to an input plane wave of amplitude $E_{0}=1$. 

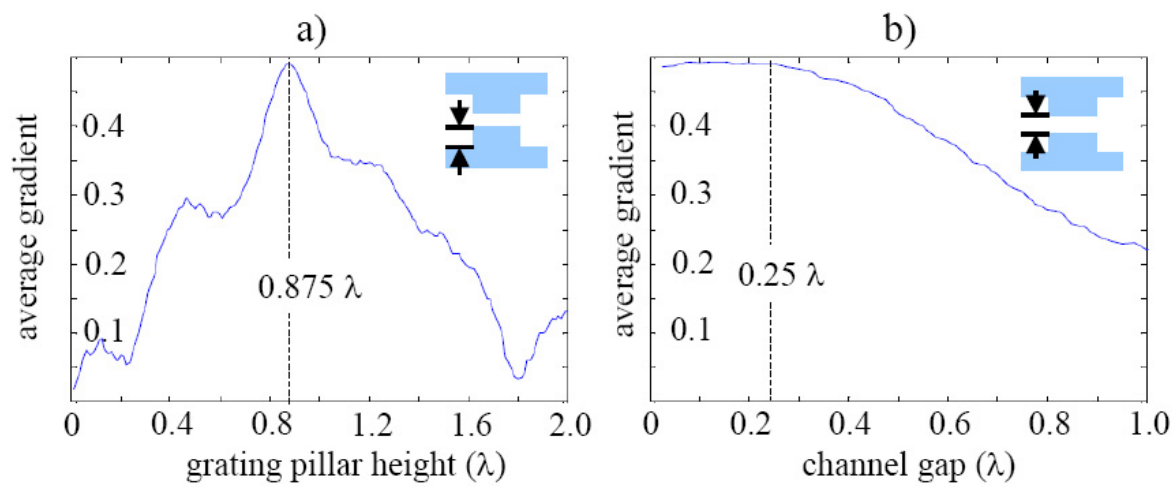

FIG. 4. (Color) (a) Average energy gradient versus grating pillar height. The value of the gradient corresponds to an input plane-wave field with an amplitude of $E_{0}=1$. (b) Average gradient versus vacuum channel gap at the narrow region of the channel. The pillar height for this plot corresponds to $0.9 \lambda$.

$$
f_{D}=\frac{G_{0}}{\left|E_{\max }\right|}
$$

For the quartz structure with grating pillars of $0.9 \lambda$, the maximum electric field is found at the center part of the narrow gap surface and as was shown in Fig. 2(b) has an absolute value of $\left|E_{\max }\right| \sim 2.05 E_{0}$. The estimated gradient of $G_{0} \sim 0.49 E_{0}$ results in a damage factor $f_{D} \sim 0.24$, which is slightly larger than the effective damage factor ratio of photonic crystal accelerators [5]. In sum, the quartz-based structure is able to convert $\sim \frac{1}{4}$ of the local largest peak electric field into an average unloaded gradient.

The damage factor of this quartz-based structure can be improved by a choice of a different material. For example, consider diamond, which has an index of $n \sim 2.36$ and shows an unusually high thermal conductivity. Figure 5(a) shows the optimum grating pillar height for such a diamond based structure occurring at $0.625 \lambda$, which is shorter than the optimum pillar height for quartz shown in Fig. 4(a).
At the pillar height of $0.625 \lambda$ the average gradient is $G_{0} \sim 0.75 E_{0}$, and the surface field is $\left|E_{\max }\right| \sim 2.18 E_{0}$, leading to a higher damage factor $f_{D} \sim 0.34$ when compared to the quartz structure. Figure 5(b) shows the average gradient versus width of the vacuum channel in the narrow part of the gap region. The optimum channel width occurs at $\sim \frac{1}{4} \lambda$, and for widths of up to $\sim \frac{1}{2} \lambda$ the gradient is not seriously compromised. The moderate improvement of the damage factor can be attributed to the fact that shorter grating pillars are required to achieve optimum phase modulation and hence the focusing effect caused by the grating pillars is partially mitigated. However, other factors such as laser-damage threshold of the material in question determine whether the modest increase of the damage factor is worthwhile.

\section{Estimate of the lateral deflection forces}

In addition to the accelerating field, lateral deflection forces play an important role in an accelerator and can potentially have a disruptive effect on the particle's trans- a)

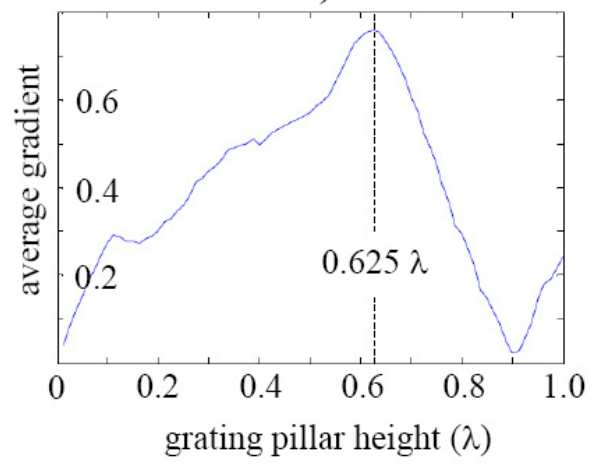

b)

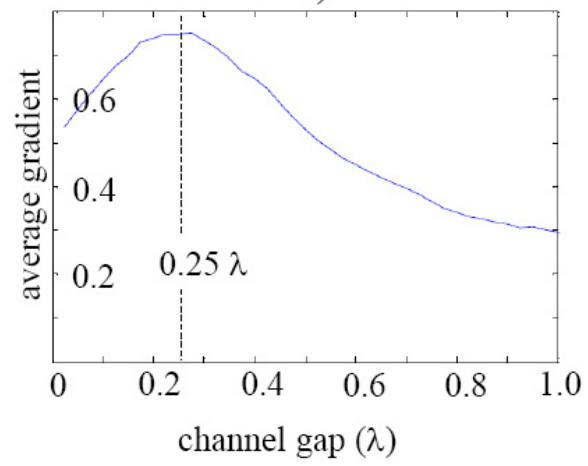

FIG. 5. (Color) Optimization of a diamond $n=2.36$ grating structure. Compare with Fig. 4 for a quartz structure. (a) Average gradient versus grating pillar height at a vacuum channel gap width of $\frac{1}{4} \lambda$. (b) Average gradient versus vacuum channel width for a pillar height of $0.625 \lambda$. 
port through the structure. It is therefore important to evaluate these force components. For the geometry described here where only $E_{x}, E_{y}$, and $B_{z}$ are present, the lateral deflection produced by the electromagnetic field is found from the Lorentz force. The deflection force is oriented in the horizontal plane and has a magnitude $F_{y}=$ $q\left(E_{y}+\beta c B_{z}\right)$. The field components $E_{x}$ and $B_{z}$ are automatically calculated by the FD-TD numerical analysis, hence no additional computation is required to find these fields along the particle's trajectory. Similar to particle acceleration of relativistic electrons from a single laser beam in vacuum, the deflection force from the electric field $\left\langle F_{E}\right\rangle$ and the deflection force from the magnetic field $\left\langle F_{H}\right\rangle$ are found to be oriented in opposite directions and are comparable in magnitude [22]. Figure 6(a) shows these force components for the crystal quartz structure described earlier. Notice that both transverse force components are close in value to the input field $E_{0}$, but have opposite sign and almost perfectly cancel each other.

The cancellation of the transverse forces is not perfect, and depending on the phase of the particle a small residual deflection force remains. However, the maximum deflection is only about $5 \%$ of the maximum gradient and occurs at a particle phase of no acceleration. This is shown in Fig. 6(b), which displays the average deflection force and average longitudinal force (the gradient) normalized to their respective maxima. Near the phase of maximum acceleration (A) or of maximum deceleration (D), the residual average deflection force $\left\langle F_{\perp}\right\rangle$ is only $\sim 0.5 \%$ of the longitudinal average force $\left\langle F_{\|}\right\rangle$. This implies that simple schemes such as occasional reversal of the sidepumping orientation of the structure or the addition of external dipole steering elements can be used to compensate the residual deflection from the laser field. Pumping of the structure from both sides completely cancels the lateral deflection forces, just as in the crossed Gaussian laser beam acceleration scheme [23] but requires an additional laser beam.

Figure 6(b) suggests that if a net deflection is indeed desired the phase of the plane wave can be moved by about $\pm \pi / 2$, the sign determining the direction of the deflection. Hence, certain sections of the grating accelerator structure could in principle be devoted as active laser-driven beam transport steering elements. However, the strongest possible laser-driven deflection will only be $\sim 5 \%$ of the maximum possible gradient. Finally, similar to wave guide accelerators, if the bunch is slightly ahead of the phase point A (the acceleration crest) it experiences longitudinal compression whereas behind the crest it can be expected to stretch. Thus, in a similar fashion to waveguide accelerators, slight optical phase adjustments to the laser can also be employed for this structure to control the longitudinal bunch compression.

\section{Ultrashort laser pulse operation}

One of the main motivations for investigating laserdriven particle accelerator structures has been their potential for sustaining a high gradient due to the laser-damage threshold properties of dielectric materials from ultrashort, near-infrared laser pulses [10]. For laser pulses below $1 \mathrm{ps,}$ the damage fluence for dielectric materials has been observed to be on the order of $\sim 2 \mathrm{~J} / \mathrm{cm}^{2}$ [24,25], implying that for a $100 \mathrm{fs}$ laser pulse the dielectric surface could sustain peak electric fields on the order of $\sim 10 \mathrm{GV} / \mathrm{m}$. The challenge for all dielectric laser-accelerator structures is to convert the largest possible fraction of that field into a synchronous longitudinal accelerating field. For the quartz structure the $\sim 10 \mathrm{GV} / \mathrm{m}$ implies that a sustained unloaded gradient of $G_{0} \sim 2.5 \mathrm{GV} / \mathrm{m}$ is possible with $100 \mathrm{fs}$ pulse durations. An interesting question is whether it is possible to drive the structure with even shorter few-cycle laser pulses and with the aim to drive the gradient to higher a)

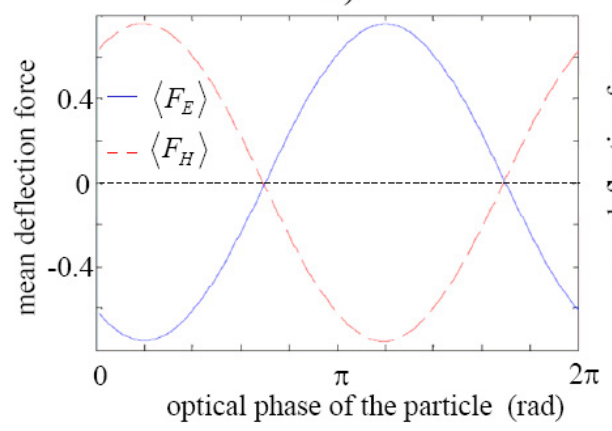

b)

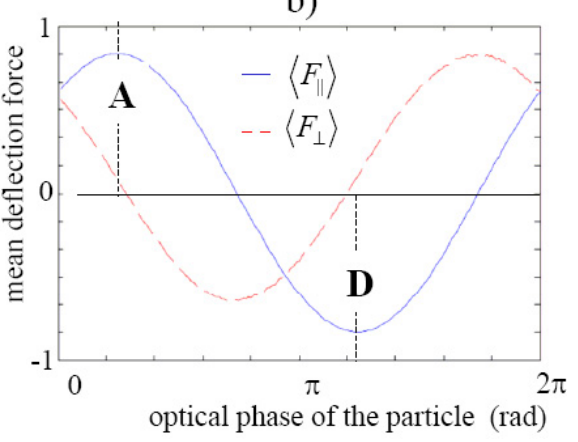

FIG. 6. (Color) (a) Plot of the magnetic $\left\langle F_{H}\right\rangle$ and electric $\left\langle F_{E}\right\rangle$ mean deflection force experienced by the particle over one accelerator period as a function of the particle launch phase. The forces are normalized to the input plane-wave field amplitude $E_{0}=1$. Hence, the maximum value of $\left\langle F_{E}\right\rangle \sim 0.6$ means that the average amplitude of the transverse electric experienced by the particle at that optical phase is $\sim 0.6 E_{0}$. (b) Plot of the total transverse mean deflection force $\left\langle F_{\perp}\right\rangle=\left\langle F_{H}\right\rangle+\left\langle F_{E}\right\rangle$ and mean longitudinal force $\left\langle F_{\|}\right\rangle$versus particle phase. Both curves of $\left\langle F_{\perp}\right\rangle$ and $\left\langle F_{\|}\right\rangle$are normalized to their respective maximum values. Phase A corresponds to the maximum acceleration phase and $\mathbf{D}$ corresponds to maximum deceleration phase. 
values. For a $10 \mathrm{fs}$ pulse $G_{0}$ would increase by $\sqrt{10}$ to $\sim 7.9 \mathrm{GV} / \mathrm{m}$.

For the diamond structure described earlier illuminated with a $10-\mathrm{fs}, 2 \mathrm{~J} / \mathrm{cm}^{2}$ laser pulse, the unloaded gradient could exceed $10 \mathrm{GV} / \mathrm{m}$. This implies that higher index dielectric materials can lead to higher gradients provided the damage threshold value is not compromised by the properties of the material in question. In addition to the index of refraction criteria, the ultimate choice of a material for the accelerator structure described here will depend on other factors such as transparency range, nonlinear optical coefficients, chemical stability, radiation hardness, thermal conductivity, and availability to name a few. With an index of $\sim 3.5$, a good thermal conductivity and availability silicon would appear to be an ideal choice; however, its transparency range requires the use of $\lambda \sim 2 \mu \mathrm{m}$ lasers that at present are not a mature technology. On the other hand, since very efficient lasers have been developed at $\lambda \sim 1 \mu \mathrm{m}$, we are more inclined to explore dielectric materials transparent at this wavelength, such as fluorides, sapphire, quartz, YAG, $\mathrm{Y}_{2} \mathrm{O}_{3}$ or even diamond as more likely substrates for a laser-accelerator structure.

Under the few-cycle laser pulse conditions, the question of possible degradation of the periodic field reversal in the vacuum channel due to the high bandwidth of the laser pulse becomes important. This question can be addressed with the FD-TD numerical analysis, which can show the evolution of the field components in time. Figure 7 shows a simulation of the passage of a two cycle 5-fs plane-wave traversing the crystal quartz structure.

Figure 7(b) displays the field as it traverses the vacuum channel, and shows that the periodic field reversal seen in the vacuum channel for cw fields [shown in Fig. 2(a)] is preserved. Figure 7(c) that no significant field remains in the vacuum channel after the passage of the pulse. This is not surprising since the vacuum channel acts as a low $Q$ resonator that has only bulk interfaces with small reflection coefficients. Therefore the buildup and dissipation of the field in that volume occurs within an optical cycle. In Fig. 7(c) it is observed that there is a small amount of reflection that could be in principle minimized by altering the gap width to a configuration where it partially functions as an antireflection layer. However, due to the high bandwidth of the pulse, the residual reflection can only be reduced but not completely eliminated. Figure 7(d) shows the instantaneous longitudinal electric field at the vacuum gap center as a function of time, confirming that the fewcycle pulse profile is preserved in the vacuum gap with only very small amount of ring-down time.

\section{E. Structure impedance, loaded gradient, and optimum efficiency}

This structure is different from waveguide accelerator geometries in that the electromagnetic energy is not copropagating with the electrons in the vacuum channel and is also different from near-field structures that resonate the a)

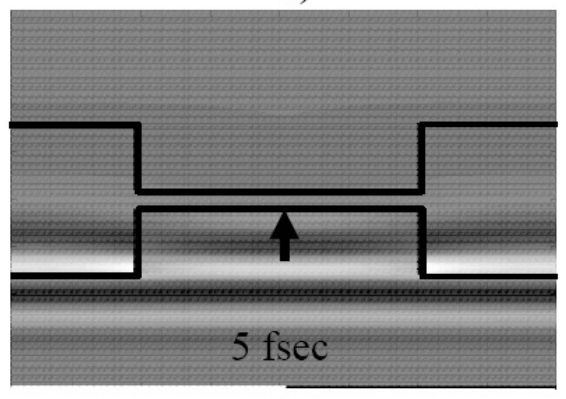

c)

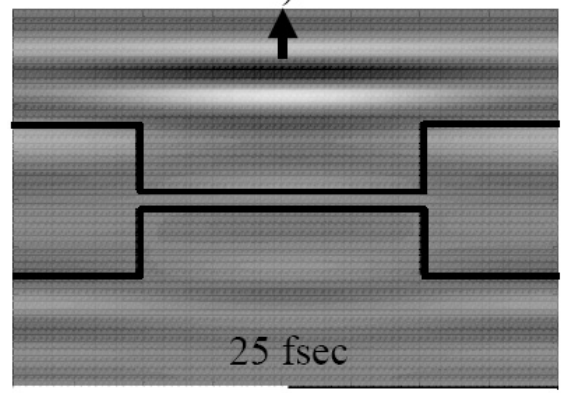

b)

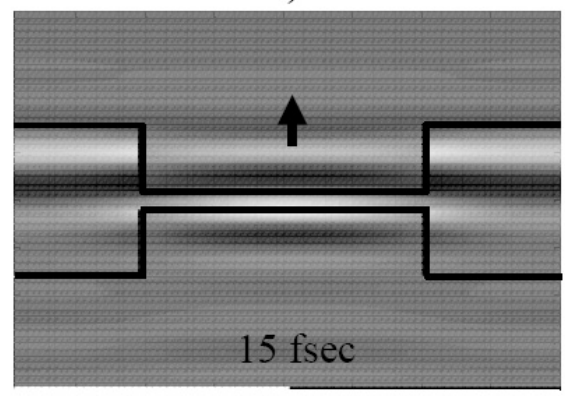

d)

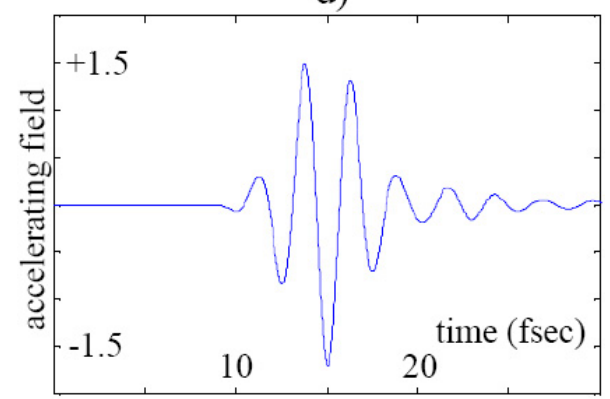

FIG. 7. (Color) (a,b,c) Propagation of a $\tau_{\text {laser }} \sim 5$ fs plane wave of center wavelength $\lambda=800 \mathrm{~nm}$ in a quartz-based grating ( $n=$ 1.55). Each frame corresponds to a $5 \mathrm{fs}$ time increment. The arrow indicates the direction of the wave. (d) Instantaneous longitudinal electric field at the vacuum gap channel center as a function of time. 
field. The characteristic impedance of the structure is defined as the ratio of the square of the voltage gained over the power lost in the structure [26]. For one grating period $\Delta z=\lambda$,

$$
Z_{c}=\frac{\left|G_{0} \Delta z\right|^{2}}{\Delta P_{\text {laser }}}
$$

For simplicity, assume a flattop intensity profile laser beam with a dimension $D$ in the vertical dimension $(z$-axis in Fig. 1). The peak power of the incoming plane wave over one grating period is

$$
\Delta P_{\text {laser }}(t)=\frac{E_{0}^{2}}{2 Z_{0}} \lambda D,
$$

where $E_{0}$ is the peak electric field amplitude and $Z_{0}=$ $377 \Omega$ the vacuum impedance. As described in Sec. II, at the optimum grating pillar height of $0.9 \lambda$ the gradient $G_{0}$ is related to the plane-wave amplitude by $G_{0} \sim \frac{1}{2} E_{0}$. Combining these expressions yields an estimate for the structure impedance of the structure that scales as

$$
Z_{S}=\frac{\lambda}{2 D} Z_{0}
$$

As suggested by Eq. (6) focusing of the laser in the vertical plane (by a cylindrical lens) improves the structure impedance. Selecting a reasonable focus that produces a vertical laser spot size of $D \sim 5 \lambda$ yields a structure impedance of $Z_{S} \sim Z_{0} / 10 \sim 37 \Omega$. This value is comparable to the structure impedance evaluated for the $2 \mathrm{D}$ photonic band gap fiber accelerator structure $Z_{S} \sim 19 \Omega$ [8]. The impedance of the structure and the applied bunch charge determine the loaded gradient experienced by the particles. Since the laser power is flowing at right angles to the electron beam, the effect of beam loading is different from that occurring with waveguide structures, as is shown in the Appendix. Assuming that the wakefield radiation loss for a single particle traversing the structure is small compared to the energy gain, the loaded gradient for a bunch with $N_{b}$ electrons is

$$
G_{L}=G_{0}-\frac{N_{b} q c Z_{S}}{2 \lambda^{2}} .
$$

At the optimum efficiency the loaded gradient is $G_{L}=$ $1 / 2 G_{0}$. For the $100 \mathrm{fs}$ laser pulse case $G_{L} \sim 1.2 \mathrm{GeV} / \mathrm{m}$, and for the few-cycle $10 \mathrm{fs}$ laser pulse $G_{L} \sim 3.9 \mathrm{GeV} / \mathrm{m}$. For the few-cycle laser pulses, it will not be feasible to accelerate more than one bunch per laser pulse, mainly because these would be located at different locations of the pulse envelope and would experience significantly different accelerating fields. The Appendix shows that assuming that only one electron bunch is present the maximum efficiency is

$$
\eta_{\mathrm{opt}}=\frac{U_{\text {cycle }}}{2 U_{\text {pulse }}}
$$

$U_{\text {cycle }}$ is the laser energy of the optical cycle where the electron bunch is present and $U_{\text {cycle }}$ is the total laser pulse energy. For a laser pulse with a Gaussian time profile $I(t)=I_{0} e^{-t^{2} / \tau_{\text {pulse }}^{2}}$, the pulse energy is $U_{\text {pulse }} \sim \sqrt{\pi} I_{0} \tau_{\text {pulse }}$ and the laser energy of the optical cycle is $U_{\text {cycle }} \sim I_{0} \tau_{\text {cylce }}$ where $\tau_{\text {cylce }}=\lambda / c$. Hence, the efficiency for a single bunch in a laser pulse is

$$
\eta_{\mathrm{opt}}=\frac{1}{2 \sqrt{\pi}} \frac{\tau_{\text {cycle }}}{\tau_{\text {pulse }}}
$$

Equation (9) shows that a few-cycle laser pulse is also beneficial from an energy efficiency point of view because it has better temporal overlap with a single bunch. For a $100 \mathrm{fs}, \lambda=800 \mathrm{~nm}$ laser pulse the maximum efficiency is only $\sim 0.8 \%$, but if $\tau_{\text {pulse }}$ is reduced to about 10 fs the efficiency climbs to about $8 \%$, which is comparable to the single bunch energy efficiency of a wave guide based laseraccelerator structure without resonating the field [27]. At the optimum efficiency of the structure, the number of electrons per bunch is given by

$$
N_{\mathrm{opt}}=\frac{G_{0} \lambda^{2}}{c q Z_{s}} .
$$

For the quartz structure, a laser wavelength of $\lambda=800 \mathrm{~nm}$ and a pulse duration of $100 \mathrm{fs}$, the optimum bunch charge corresponds to $\sim 9 \times 10^{5}$ electrons. Note that the gradient appears in the numerator of Eq. (10). Once again, with a shorter laser pulse of $10 \mathrm{fs}$ the unloaded gradient increases by a factor of $\sqrt{10}$ and $N_{\text {opt }} \sim 3 \times 10^{6}$. This is a significantly larger number of electrons than the charge per bunch supported by the waveguide based geometries.

Intuitively, one might explore enclosing the grating by another resonator microstructure encompassing the gap to significantly improve the efficiency. A resonator based geometry would allow for an enhancement of the accelerating field in the cavity but at the same time would require a filling time that is proportional to the $Q$ of the cavity. The extended presence of the laser field in the structure would compromise the ability to support high-peak electric fields below the damage threshold and the whole advantage for employing ultrashort laser pulses would be lost. Furthermore a high- $Q$ cavity has a low bandwidth and would couple very poorly with an ultrashort laser pulse. Potentially the accelerator structure could be included as an intracavity element of an external macroscopic resonator with a mode spacing that matches the laser repetition rate, in a similar fashion as proposed for wave guide laserdriven accelerators [27]. However, to support the high bandwidth from an ultrashort laser the external cavity would require higher-order dispersion compensation elements. The structure described here is ideally a single-pass high-bandwidth element to be powered with ultrashort laser pulses, and not a high- $Q$ resonator based accelerator 
structure. A final possibility for recycling the laser power is to reimage the laser between adjacent structures and produce an array of electron beams. In this instance, the structure geometry would have to be optimized for maximum transmission of the plane wave through the vacuum channel. However, the complications arising from such a scheme may outweigh the benefits and further investigation of this idea will be necessary to assess its feasibility.

\section{F. Power consumption and thermal loading considerations}

One of the historical motivations for investigating laserdriven particle acceleration concepts has been its potential for use in a future $\mathrm{TeV} e^{+} e^{-}$collider. One of the main challenges presented to laser-driven particle acceleration is the desired beam luminosity for such a collider, on the order of $\sim 10^{38} / \mathrm{m}^{2}$. Hence, if the accelerator structure presented here is to be applied in a future $\mathrm{TeV} e^{+} e^{-}$ collider it becomes important to seek the parameter space that satisfies the luminosity requirement. The argument that follows is presented as a preliminary order-ofmagnitude estimate to assess the overall feasibility for the double-grating laser-accelerator structure from the luminosity perspective. The luminosity at the collision point (IP) of two beams has the form

$$
L \sim \frac{f_{\text {rep }} N_{b}^{2}}{4 \pi \sigma_{x} \sigma_{y}}
$$

where $f_{\text {rep }}$ is the electron bunch repetition rate, $N_{b}$ the number of electrons per bunch and $\sigma_{x} \sigma_{y}$ are the transverse spot sizes at the IP. The optimum number of electrons per bunch for the accelerator structure described here was found to be $N_{b} \sim 10^{6}$, which is 3 orders of magnitude lower than the design $\sim 1 \mathrm{nC}$ bunch charge for the next rf-technology based $\mathrm{TeV}$ collider. To maintain the luminosity, the lower bunch charge has to be compensated with a smaller electron beam spot size at the IP and a higher electron bunch repetition rate to gain back 6 orders of magnitude. Because of the significantly lower transverse emittance and lower bunch charge expected for structure loaded laser-driven particle accelerators, IP spot sizes on the order of $\sim 1 \mathrm{~nm}$ are potentially feasible. Therefore, to reach $L \sim 10^{38} / \mathrm{m}^{2}$ the required repetition rate for this laser-accelerator structure is $\sim 100 \mathrm{kHz}$, which lies well within the typical mode-locked laser repetition rates. If the structure is to be operated at the desired $\mathrm{GeV} / \mathrm{m}$ gradients near the damage threshold fluence $\sim 1 \mathrm{~J} / \mathrm{cm}^{2}$ at a $100 \mathrm{kHz}$ repetition rate, it will require an average laser power density of about $10^{5} \mathrm{~W} / \mathrm{cm}^{2}$. For the suggested laserfocusing spot size of $\sim 5 \mu \mathrm{m}$ about $10 \mathrm{~kW}$ of laser power per meter are required. Assuming that each laser amplifier section can produce $100 \mathrm{~W}$ of average power, the structure will require one laser for every $\mathrm{cm}$ of accelerator structure.
At these laser power levels, thermal loading and heat management cannot be overlooked. First, consider the heat produced by the wakefields of the electron beam. The energy loss gradient in a dielectric structure with a vacuum channel having an aperture size $R$ is approximately $G_{H} \sim$ $q c Z_{0} / R^{2}$ and varies by factors of unity depending on the geometry of the aperture [28]. With $R \sim \frac{1}{4} \lambda$ the energy loss gradient is on the order of $G_{H} \sim 100 \mathrm{keV} / \mathrm{m}$. Therefore the approximate energy lost per bunch $\left(N_{b} \sim 10^{6}\right)$ per meter structure due to wakefields is $\sim 10^{-8} \mathrm{~J} / \mathrm{m}$. At the quoted bunch repetition rate this corresponds to an average power of $\sim 1 \mathrm{~mW} / \mathrm{m}$. Therefore even if the entire wakefield power was converted to heat the thermal load from the electron beam is negligible.

Next, consider heat produced in the structure by absorption of laser power from impurities in the transparent medium. Even high optical quality materials have impurities that can deposit heat from the laser into the medium. For example, commercial quartz has an absorption coefficient on the order of $\alpha_{A} \sim 10^{-5} / \mathrm{cm}$. Assuming the laser beam has to traverse a $1 \mathrm{~mm}$ wide structure, this would lead to a heat production of $\sim 10 \mathrm{~mW} / \mathrm{m}$, which can also be neglected. However, the electrical power required to produce the $10 \mathrm{~kW} / \mathrm{m}$ of laser power generates the largest amount of heat. Assuming a high-power mode-locked laser system that reaches $50 \%$ wall plug efficiency about $10 \mathrm{~kW} / \mathrm{m}$ of heat are generated from the high-power laser gain medium itself. Therefore to minimize thermal loading from this source to the accelerator it is desirable to design the laser system as a physically separated object from the accelerator structure. Assuming the amplifier section of the laser system is a $\sim 1 \mathrm{~cm}$ long slab similar in geometry to those used in high-power $\mathrm{cw}$ laser amplifiers about $100 \mathrm{~W} / \mathrm{cm}^{2}$ of heat per unit area have to be removed from its surface. This lies well within the capability of silicon micro channel coolers [17].

\section{PROPOSED EXPERIMENTS}

\section{A. Fabrication and optical characterization}

The structure can be fabricated with existing nanofabrication technology. One avenue for manufacturing the double-grating structure is to produce two separate gratings and to stack them with spacers. The fabrication of the gratings can be accomplished by different methods. For example, we have selected to utilize e-beam lithography and reactive ion etching to manufacture precision binary gratings. Figure 8 shows an electron microscope photograph of a $1 \mu \mathrm{m}$ period grating structure fabricated by reactive ion etching.

\section{B. Proposed electron beam experiment setup}

We plan to perform the first test with electron beam at the E163 facility at SLAC, utilizing the available $60 \mathrm{MeV}$ electron beam and a $\sim 200$ fs pulse Ti:sapphire based laser 


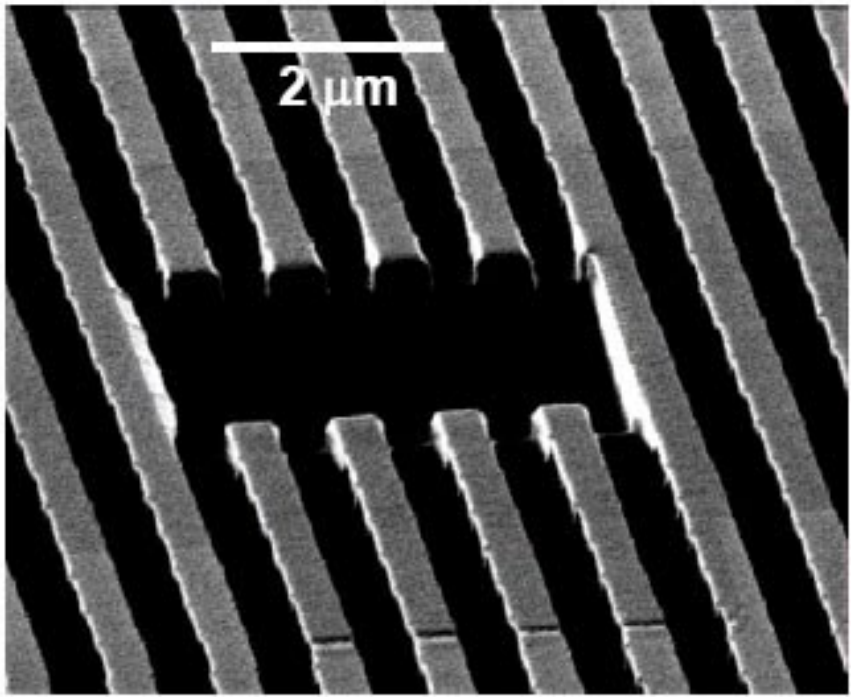

FIG. 8. Electron microscope picture of a fused silica grating with a period of $1 \mu \mathrm{m}$. The hole in the center was cut to expose the surface profile of the grating.

system. We plan to test an $8 \mathrm{~mm}$ long quartz-based accelerator cell with a variable vacuum gap. The laser beam will be pulse-front tilted at $45^{\circ}$ and focused with a cylindrical lens to a $100 \mathrm{~mm} \times 1 \mathrm{~cm}$ spot. With $\frac{1}{2} \mathrm{~mJ} /$ pulse available at the experiment, it will produce a fluence of $\sim 50 \mathrm{~mJ} / \mathrm{cm}^{2}$ and a corresponding plane-wave electric field amplitude of $\sim 1.4 \mathrm{GV} / \mathrm{m}$. Under optimum conditions, we expect the structure to produce an average gradient of $\sim 0.7 \mathrm{GeV} / \mathrm{m}$ and a corresponding maximum energy gain of $\sim 7 \mathrm{MeV}$. These laser-focusing values are well below the damage fluence and tighter focusing for higher gradients will be possible in principle. The challenge for the experiment is the loss of electron beam through the narrow, cm-long vacuum channel. Comparable electron beam transport conditions were encountered at the original LEAP experiment, where the electron beam was successfully transmitted through the $\sim 5$ micron wide aperture of a few-mm long dielectric cell [29].

The proposed setup is shown in Fig. 9. The alignment and spacing between the two gratings will be monitored with an alignment laser illuminating a flat region of the two quartz plates. One grating will be fixed while the other will be controlled by a translation stage for variable gap width.

\section{CONCLUSION}

The calculations presented in this article suggest that the proposed double-grating structure could be a feasible laser accelerator with the possibility for loaded gradients greater than $1 \mathrm{GeV} / \mathrm{m}$. Furthermore, the structure does not suffer from group-velocity limitations and therefore each segment could be several $\mathrm{mm}$ in length. Each segment can be powered by a single laser plane-wave and requires no laser mode converters. Finally, the simplicity of the device and its ease of fabrication are a strong motivation for investigating this geometry further and to perform tests with electron beam.

\section{ACKNOWLEDGMENTS}

This work is supported by DOE Grant No. DE-FG0203ER41276.

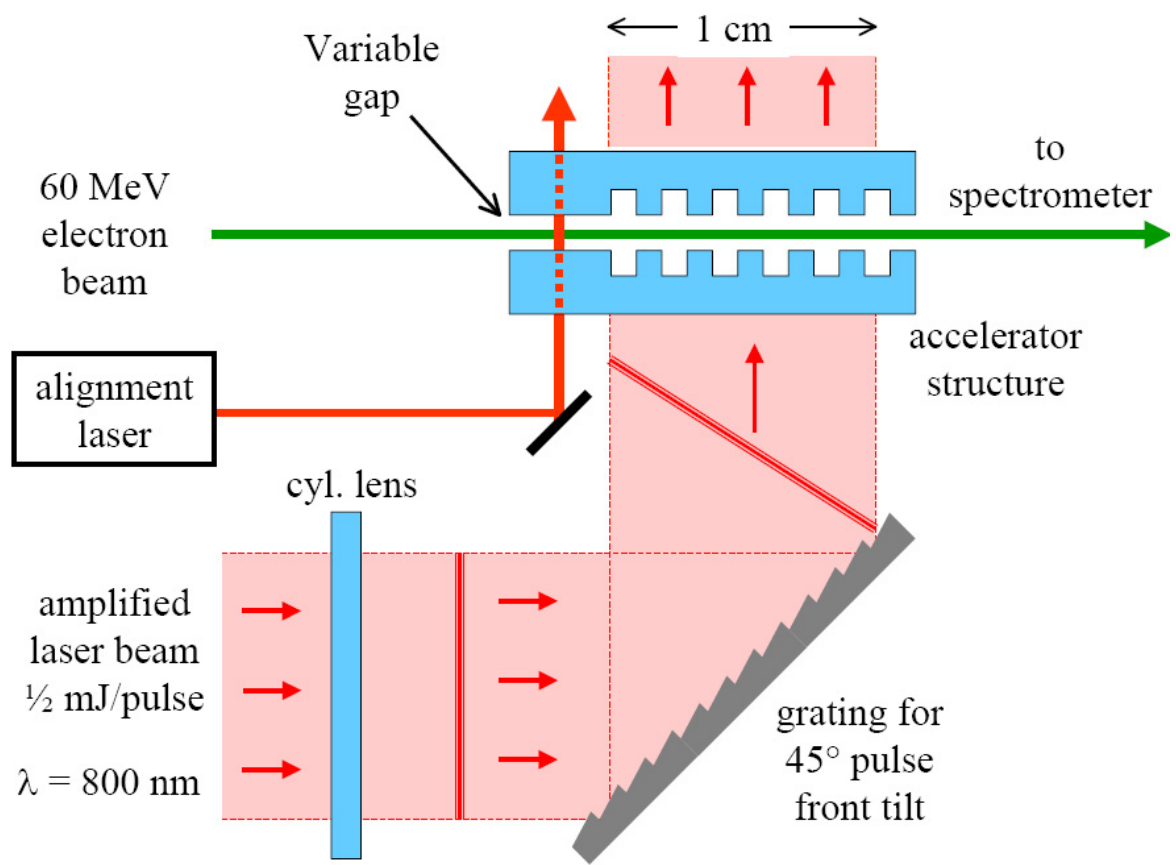

FIG. 9. (Color) The proposed setup for beam experiments with the double-grating structure. 


\section{APPENDIX: BEAM LOADING OF THE STRUCTURE}

The effect of beam loading for this type of structure can be calculated using an energy balance argument similar to that employed in waveguide based accelerators [30]. The fundamental difference to wave guide accelerators is that the electromagnetic energy is not flowing with the electron beam.

We assume a plane wave of amplitude $E_{0}$ incident on the structure and producing an unloaded average gradient $G_{0}$. Assume first that a single electron is traveling a distance $L$ in a transit time $\tau_{c}=L / \beta c$ in the structure. By Poynting's theorem, the energy change of the electron $q V_{1}$ over that distance has to be equal to the electromagnetic energy absorbed from the incoming plane wave:

$$
q V_{1}=-\frac{1}{\mu_{0}} \int_{0}^{\tau_{c}} \oint_{S}(\vec{E} \times \vec{B}) \hat{n} d t .
$$

Equation (A1) assumes that no electromagnetic energy is stored in the volume of interest, which is a very good approximation for this structure. $\vec{E}$ and $\vec{B}$ are the total instantaneous electric and magnetic fields, and they are a linear superposition of the particle's wakefields and the driving field produced from the laser. The total electric field amplitude can be described by $E=E_{L}+E_{W}+E_{C H}$, where $E_{L}$ is the laser field, $E_{W}$ the particle's wakefield that overlaps with the laser field, and $E_{C H}$ the nonoverlapping component of the particle's wakefield. In Sec. IIF it is found that for the chosen bunch charge $E_{C H}$ is $\sim 100 \mathrm{keV} / \mathrm{m}$ and hence small in comparison to the accelerating gradient. Therefore we will neglect it from here on. Finally, we assume that the electromagnetic fields enter and leave the structure only through the vertical walls and neglect fields copropagating with the electron beam. For the length of the section of interest $L$ and time $\tau_{c}$, the laser field has fluence that corresponds to an average electric field amplitude $E_{W}$ with an effective area $A$ acting over an effective time $\tau_{c}$. This allows us to evaluate the electron's energy gain expression of (A1):

$$
q V_{1}=-\frac{A}{Z_{0}}\left(E_{W}^{2}+E_{L} E_{W}\right) \tau_{c} .
$$

The overlapping field component $E_{W}$, by definition, is proportional to the laser field amplitude $E_{L}$ and therefore $E_{W}=-\alpha E_{L}$, where we assume that $|\alpha| \ll 1$. For a single electron the energy change is

$$
q V_{1} \sim \alpha \frac{A \tau_{c}}{Z_{0}} E_{L}^{2}
$$

We can express the constant $\alpha$ in terms of the shunt impedance $Z_{S}=V^{2} / P_{L}$ where the laser power is $P_{L}=$ $A E_{L}^{2} / 2 Z_{0}$ :

$$
\alpha=\frac{q Z_{S}}{2 V_{1} \tau_{c}} .
$$

When loading the structure with a large number of electrons the longitudinal wakefield components add coherently and Eq. (A2) modifies to

$$
N q V_{N}=-\frac{A}{Z_{0}}\left(N^{2} E_{W}^{2}+N E_{L} E_{W}\right) \tau_{c},
$$

where $N$ is the number of electrons and $V_{N}$ is the new voltage under beam loading conditions. Using expression (A3) the new voltage is $V_{N}=V_{1}(1-N \alpha)$ and therefore the loaded gradient is

$$
G_{L}=G_{0}-\frac{N q \beta c Z_{S}}{2 \lambda^{2}} .
$$

The efficiency is the ratio of the particle's energy gain to the applied laser energy. For $N$ particles and a loaded gradient $G_{L}$, the efficiency is

$$
\eta(N)=\frac{N q L}{P_{L} \tau_{c}} G_{L}(N) .
$$

Equation (A7) is found to have an optimum value of $\eta=\frac{1}{2}$ where the loaded gradient $G_{L}$ corresponds to $\frac{1}{2} G_{0}$ and the number of electrons is

$$
N_{\mathrm{opt}}=\frac{G_{0} \lambda^{2}}{\beta c q Z_{S}} .
$$

If there is only one electron bunch, the efficiency scales down with the ratio of the optical cycle to the laser pulse duration

$$
\eta_{\mathrm{opt}}=\frac{1}{2 \sqrt{\pi}} \frac{\tau_{\text {cycle }}}{\tau_{\text {pulse }}}
$$

[1] R. Ell, U. Morgner, F. X. Krtner, J. G. Fujimoto, E. P. Ippen, V. Scheuer, G. Angelow, T. Tschudi, M. J. Lederer, A. Boiko, and B. Luther-Davies, Opt. Lett. 26, 373 (2001).

[2] H. R. Telle, G. Steinmeyer, A. E. Dunlop, J. Stenger, D. H. Sutter, and U. Keller, Appl. Phys. B 69, 327 (1999).

[3] D. Jones, S. Diddams, J. Ranka, A. Stentz, R. Windeler, J. Hall, and S. Cundiff, Science 288, 635 (2000).

[4] J. Reichert, M. Niering, R. Holzwarth, M. Weitz, Th. Udem, and T.W. Hänsch, Phys. Rev. Lett. 84, 3232 (2000).

[5] J. Rauschenberger, T. M. Fortier, D. J. Jones, J. Ye, and S. T. Cundiff, Opt. Express 10, 1404 (2002).

[6] T. M. Fortier, D. J. Jones, J. Ye, and S. T. Cundiff, Opt. Lett. 27, 1436 (2002).

[7] R. K. Shelton, L.-S. Ma, H. C. Kapteyn, M. M. Murnane, J. L. Hall, and J. Ye, Science 293, 1286 (2001).

[8] X. Eddie Lin, Phys. Rev. ST Accel. Beams 4, 051301 (2001).

[9] B. M. Cowan, Phys. Rev. ST Accel. Beams 6, 101301 (2003).

[10] Y.C. Huang, D. Zheng, W. M. Tulloch, and R. L. Byer, Appl. Phys. Lett. 68, 753 (1996). 
[11] R. B. Yoder and J. B. Rosenzweig, Phys. Rev. ST Accel. Beams 8, 111301 (2005).

[12] J. Rosenzweig, A. Murokh, and C. Pellegrini, Phys. Rev. Lett. 74, 2467 (1995).

[13] J. D. Lawson, IEEE Trans. Nucl. Sci. 26, 4217 (1979).

[14] R. B. Palmer, SLAC-PUB-4161, 1986.

[15] J. Hebling, Opt. Quantum Electron. 28, 1759 (1996).

[16] Zs. Bor and B. Racz, Opt. Commun. 54, 165 (1985).

[17] R. L. Byer, Science 239, 742 (1988).

[18] M. K. Reed and R. L. Byer, Proc. SPIE Int. Soc. Opt. Eng. 1021, 128 (1988).

[19] R. J. Shine, A. J. Alfrey, and R. L. Byer, Opt. Lett. 20, 459 (1995).

[20] Karl S. Kunz and Raymond J. Luebbers, The Finite Difference Time Domain Method for Electromagnetics (CRC Press, Boca Raton, 1993).

[21] Ulf Anderson, Doctoral dissertation, Royal Institute of Technology, Stockholm, 2001.

[22] T. Plettner, R. L. Byer, E. Colby, B. Cowan, C. M. S. Sears, J. E. Spencer, and R. H. Siemann, Phys. Rev. ST Accel. Beams 8, 121301 (2005).
[23] E. Esarey, P. Sprangle, and J. Krall, Phys. Rev. E 52, 5443 (1995).

[24] B. C. Stuart, M. D. Feit, S. Herman, A. M. Rubenchik, B. W. Shore, and M.D. Perry, J. Opt. Soc. Am. B 13, 459 (1996).

[25] D. Du, X. Liu, G. Korn, J. Squier, and G. Mourou, Appl. Phys. Lett. 64, 3071 (1994).

[26] Thomas P. Wangler, Principles of RF linear Accelerators (John Wiley \& Sons, Inc., New York, 1998), p. 47.

[27] Y. C. Neil Na, R. H. Siemann, and R. L. Byer, Phys. Rev. ST Accel. Beams 8, 031301 (2005).

[28] R. H. Siemann, Phys. Rev. ST Accel. Beams 7, 061303 (2004).

[29] R. L. Byer, T. Plettner, Y. C. Huang, E. Colby, R. L. Siemann, J.E. Spencer, H. Wiedemann, C. Barnes, T. I. Smith, and R.L. Swent, The Laser Driven Electron Accelerator Experiment at Stanford University, Proceedings of the 1999 Particle Accelerator Conference, New York, 1999, pp. 321-324.

[30] K. L.F. Bane and G. Stupakov, Phys. Rev. ST Accel. Beams 6, 024401 (2003). 